\title{
The effect of agility exercise on eicosanoid excretion, oxidant status, and plasma lactate in dogs
}

\author{
Wendy I Baltzer ${ }^{\text {* }}$, Anna M Firshman², Bernadette Stang ${ }^{1}$, Jennifer J Warnock ${ }^{1}$, Elena Gorman ${ }^{1}$
} and Erica C McKenzie ${ }^{1}$

\begin{abstract}
Background: The objective was to determine the effects of agility exercise on dogs of different skill levels with respect to urinary eicosanoids, urinary $15 \mathrm{~F}_{2 \mathrm{t}}$-isoprostane (lipid peroxidation marker) and hematological/biochemical changes in plasma. Fifteen adult dogs had blood and urine samples obtained prior to, immediately and 4-hours following an agility exercise.

Results: Hematocrit, red blood cells (RBC), albumin, and hemoglobin increased following exercise, with greatest increases correlating to increased skill group (novice, intermediate, masters); at 4-hours post-exercise, hematocrit, RBC, and hemoglobin were decreased. Phosphorus increased following exercise with the greatest increase in novice and intermediates. Plasma lactate increased 3.6-fold in masters, 3.2-fold in intermediates, and 1.2-fold in novice dogs. Urine thromboxane $B_{2}$ (TXB2) more than tripled 4-hours post-exercise while 6-keto prostaglandin $F_{1 a}$ (PGF1a, prostacyclin metabolite), prostaglandin $\mathrm{E}_{2}$ metabolites (13,14-dihydro-15-keto-prostaglandin $\mathrm{A}_{2}$ and 13 , 14-dihydro-15-keto-prostaglandin $\mathrm{E}_{2}$ ), and 13,14-dihydro-15-keto prostaglandin $\mathrm{F}_{2 a}$ were unaffected as determined by a competitive enzyme immunoassay and standardized by division with urine creatinine. Urine $15 \mathrm{~F}_{2 \mathrm{t}}$-isoprostane increased insignificantly.

Conclusions: Alterations in the plasma post-exercise were likely due to hemoconcentration from insensible water loss, splenic contraction and sympathetic stimulation while 4-hours later autohemodilution reduced RBC parameters. Elevations in plasma lactate and urinary TXB2 correlated with advanced skill level/speed of the dogs.
\end{abstract}

Keywords: Agility exercise, Dog, Urinary isoprostane, Plasma lactate, Oxidant stress, Urinary eicosanoids, Thromboxane $B_{2}$

\section{Background}

The United States Dog Agility Association (USDAA) alone, registers over 25,000 competitors and organizes over 400 days of agility competitions each year in the United States, Puerto Rico, Canada, Mexico, Bermuda, and Japan [1]. The percentage of dogs injured during agility exercise is estimated at 33\% [2]. With such a high rate of injury, determining the effects of this type of exercise on the physiologic responses of dogs including

\footnotetext{
* Correspondence: wendy.baltzer@oregonstate.edu

'Departments of Clinical Sciences (Baltzer, Firshman, Stang, Warnock, McKenzie) and Biomedical Sciences (Gorman), College of Veterinary Medicine, Oregon State University, Corvallis, OR 97331, USA

Full list of author information is available at the end of the article
}

the degree of oxidant stress and eicosanoid production induced by this exercise, is important to understanding why injury rates are so high.

Canine agility exercise has been shown to induce significant increases in blood lactate and hence an anaerobic metabolic state [3,4]. In addition, plasma chloride, hematocrit, and triglycerides increase with agility exercise whereas, serum albumin, calcium and phosphorus concentrations decrease immediately after this exercise $[3,4]$. No alteration in plasma protein, sodium, potassium or creatinine concentrations has been found with agility exercise in dogs [4]. The alterations that do occur have been attributed to splenic contraction in addition to increased movement of fluid from the interstitium into the vascular

\section{Ciomed Central}


space, and subsequently into contracting muscle cells [4]. Therefore, there is an overall minimal change in plasma volume in dogs during agility exercise but a tremendous necessity for anaerobic metabolism. A wide variety of dog breeds participate in agility events, and there are a wide variety of competition levels in the sport. Hence, due to differences in fitness and innate athletic ability, some dogs may experience greater effects of exertion than others during physical activity in agility. The effect of skill and intensity level on specific physiologic variables in agility competition dogs is unknown.

Exercise increases the rate of oxygen consumption, subsequently resulting in the production of free radicals or reactive oxygen species. The reactive oxygen species cause lipid peroxidation and cell injury, which may overwhelm cellular antioxidant mechanisms [5]. In humans, intense exercise induces acute oxidant stress, reflected by increased $15 \mathrm{~F}_{2 \mathrm{t}}$ isoprostane excretion in urine [6-9]. Isosprostanes form when arachidonic acid reacts with reactive oxygen species in cell membranes and they are considered a marker of oxidant activity and lipid peroxidation in vivo in humans and several other species [10]. The period of time required for recovery from exerciseinduced oxidant damage is unknown, but in humans, isoprostane production returned to baseline within 24 hours following exercise [11,12]. In sled dogs undergoing endurance exercise, plasma isoprostanes increase following exercise and correlate with the logarithm of serum creatinine kinase activity, a marker of muscle damage [13]. To the authors' knowledge, the excretion of $15 \mathrm{~F}_{2 \mathrm{t}}$ isoprostanes in the urine of dogs following agility exercise has not been previously reported nor have hematological and biochemical parameters been followed for 4-hours post agility exercise and compared between skill groups.

Exercise in horses and humans induces an inflammatory response and has been shown to alter eicosanoid production $[14,15]$. Dogs experience an anaerobic metabolic state during agility exercise with increases in plasma lactate, hemoglobin, hematocrit, and red blood cell count, however to the authors' knowledge, the effects of agility exercise on eicosanoid production in dogs of different skill levels has not been previously investigated [3].

The purpose of this study was to determine the effects of agility exercise on dogs of different skill levels with respect to $15 \mathrm{~F}_{2 \mathrm{t}}$ isoprostane, thromboxane $\mathrm{B}_{2}$ (TXB2), 6-keto prostaglandin $\mathrm{F}_{1 \alpha}$ (PGF1 $\alpha$, prostacyclin metabolite), prostaglandin $E_{2}$ metabolites (13,14-dihydro-15-keto-prostaglandin $\mathrm{A}_{2}$ and 13,14-dihydro-15-keto-prostaglandin $\mathrm{E}_{2}$ ), and 13,14-dihydro-15-keto prostaglandin $\mathrm{F}_{2 \alpha}$ excretion in the urine and systemic hematological and biochemical changes in the plasma; and to determine if alterations in these parameters had resolved within 4 hours of the high intensity exercise.

\section{Methods}

Animals

The study protocol was approved by the Oregon State University Animal Care and Use Committee and written informed consent was obtained from all owners of dogs participating in the study prior to participation of the dogs. A local agility dog club was contacted and volunteers were requested to participate in the study. Dogs were reported by their owners to be competitors in agility either at the novice (referred to as starters by the USDAA), intermediate (advanced), or masters classes. Dogs earn entry into each class through competition and their scores in those competitions [1]. Owners reported how often their dogs trained on a weekly basis and if their dogs were currently receiving any medications. The sex, age and breed of each dog were also recorded. Each dog was examined by a veterinarian prior to participating in the agility exercise during which the heart and lungs were auscultated and their gait was examined for signs of lameness or dysfunction. Dogs were excluded from the study if they were receiving electrolyte supplements, exhibited signs of lameness the day of the experiment, or had an elevated body temperature prior to beginning the experiment $\left(\geq 103.0^{\circ} \mathrm{F}, 39.4^{\circ} \mathrm{C}\right)$. Dogs were not allowed to eat within 3 hours prior to the agility test but they were allowed training treats in small amounts following the test. A full meal was not given to any dog until after the 4-hour sample collection was performed. At no time was water consumption restricted from the dogs during the assessment.

\section{Agility exercise test}

Prior to the agility test, a heart rate monitor (S625x, Polar Electro Inc, Lake Success, NY) was placed around the girth of each dog using an elastic harness. Each dog ran a course, consisting of 11 jumps and 4 tunnels, 3 times with a 5 minute rests period between the first and second, then second and third runs. The dogs ran the course three times to duplicate the exercise they would experience during an agility competition. The height of the jumps was adjusted for each dog with dogs less than 12 inches in height at the shoulder jumping 12 inch tall jumps, dogs 12 to 16 inches tall jumping 16 inch tall jumps, dogs 16 to 21 inches tall jumping 22 inch tall jumps and dogs over 21 inches jumping 26 inch tall jumps [1]. Each dog ran the course 3 times in succession with a 5-minute rest period between runs one and two and two and three. Each dog was allowed to sit, lie down, or walk during the rest period, but not trot or run. Each run was timed in seconds with a stopwatch and the times recorded for each dog. The number of missed jumps or obstacles taken out of sequence was also recorded for each dog. Heart rate, respiratory rate, and rectal temperature were recorded 
prior to, immediately following and 4 hours following the 3 runs through the agility course.

\section{Sample collection}

Blood was collected from each dog within 2 hours prior to participating in the agility exercise, and within 2 minutes of completing the agility exercise test, and 4-hours after completing the test via jugular venipuncture. Blood was placed into EDTA and lithium heparin tubes immediately following collection, and heparinized blood was immediately submitted for determination of venous blood lactate using an automated blood gas analyzer (RapidLab 1265, Siemens Healthcare Diagnostics, Tarry Town, NY) for determination of venous blood lactate. Packed cell volume and total protein were determined immediately following collection. Hematological analyses were performed using the ADVIA 120 hematology analyzer (Siemens, Deerfield, IL), and biochemical parameters with the Hitachi 917 chemistry analyzer (Roche Group, Tucson. AZ). All hematological and biochemical analyses were performed within 4 hours of sample collection. Urine was collected from each dog by free catch technique within 2 hours prior to the agility exercise test, within 1 hour following the test, and within one-half hour of 4 hours following the exercise test. Urine was stored within 1 hour of collection at $-80^{\circ} \mathrm{C}$ until analysis.

\section{Isoprostane determination}

Samples were analyzed in duplicate and in batches according to the manufacturer's instructions to reduce interassay variation. The isoprostane assay and urinary creatinine assays have undergone extensive analytic validation by the manufacturers, as indicated in the results provided in the package insert. Urine $15 \mathrm{~F}_{2 \mathrm{t}}$ isoprostane (ISO) was determined using a competitive enzyme immunoassay $\left(15 \mathrm{~F}_{2 \mathrm{t}}\right.$ isoprostane enzyme immunoassay kit, Cayman Chemical Co, Ann Arbor, Mich) after sample pretreatment with $\beta$-glucuronidase (Oxford Biochemical Research, Oxford, MI). A spectrophotometric plate reader was used at $420 \mathrm{~nm}$. The specificity of the EIA is reported by the manufacturer as $100 \%$ cross reactivity for 8-isoprostane and 8-iso prostaglandin $F_{2 \alpha}$ ethanolamide, $20.6 \%$ for 8 -iso prostaglandin $F_{3 \alpha}$, and $4 \%$ for 2,3-dinor-8-iso porstaglandin $\mathrm{F}_{2 \alpha}$. Samples were analyzed in duplicate and in batches to reduce interassay variation. This assay has undergone extensive analytic validation by the manufacturers, as indicated in results provided in the package insert. Intra-assay coefficient of variation ranged from 19.9: at $2 \mathrm{pg} / \mathrm{ml}$ to $12.6 \%$ at $500 \mathrm{pg} / \mathrm{ml}$ and inter-assay coefficient of variation ranged from $9.6 \%$ at $2 \mathrm{pg} / \mathrm{ml}$ dose to $10.5 \%$ at $500 \mathrm{pg} / \mathrm{ml}$. In-house validation was performed with urine samples previously collected from healthy dogs as part of unrelated, previously published research in dogs [16]. Our previous validation of the assay included the assessment of dilutional parallelism of canine urine samples (5 samples at 3 dilutions in which we determined the correct dilution for samples such that they would be within the working range of the assay) and determination of interassay variability (5 samples assayed on 5 separate days) [16].

\section{Urinary eicosanoid determination}

Urine samples were kept at $-20^{\circ} \mathrm{C}$ until analysis for their prostanoid content. Thromboxane $\mathrm{B}_{2}$ (TXB2), 11-dehydro-thromboxane $B_{2}$ (11-TXB2), 6-keto prostaglandin $\mathrm{F}_{1 \alpha}$ (PGF1 $\alpha$, prostacyclin metabolite), prostaglandin $\mathrm{E}_{2}$ metabolites (13,14-dihydro-15-keto-prostaglandin $A_{2}$ and 13,14-dihydro-15-keto-prostaglandin $E_{2}$ ), and 13,14-dihydro-15-keto prostaglandin $F_{2 \alpha}$ (PGF2 $\alpha$ ) are not species-specific compounds and urinary excretion was determined using $\mathrm{ACE}^{\mathrm{TM}}$ competitive enzyme immunoassay (Cayman Chemical, Ann Arbor, MI) per the manufacturer's instructions. Samples were analyzed in duplicate and batched to reduce interassay variation. The TXB2 and 11-TXB2 assays have undergone extensive analytic validation by the manufacturers and use of 11-TXB2 in canine urine has been validated previously [17]. The PGF1 $\alpha$ assay for prostacyclin excretion has undergone extensive analytic validation by the manufacturers and with cross reactivity to prostaglandin $\mathrm{D} 2<0.01 \%$, prostaglandin $\mathrm{F} 2 \alpha=11 \%$, and TXB2 $=0.05 \%$. The prostaglandin E2 metabolite assay used is recommended for analysis of urine for metabolites of PGE2 since PGE2 is rapidly converted in vivo to its 13,14-dihydro-15-keto metabolite which is unstable and rapidly degraded to 13,14dihydro-15-keto $\mathrm{PGA}_{2}$. The assay used to determine 13,14-dihydro-15-keto-prostaglandin $\mathrm{A}_{2}$ and 13,14-dihydro15-keto-prostaglandin $E_{2}$ concentrations in the urine and has been validated by the manufacturer with crossreactivity to prostaglandin $\mathrm{D} 2$, prostaglandin $\mathrm{F} 2 \alpha$, 6-ketoprostaglandin $\mathrm{F} 1 \alpha$, prostaglandin $\mathrm{F} 1 \alpha$, and TXB $2<0.01 \%$. The PGF2 $\alpha$ assay has been used for direct quantitation of 13,14-dihydro-15-keto prostaglandin $F_{2 \alpha}$ in urine and the manufacturer has performed extensive analytical validation of the assay with cross reactivity for prostaglandin D2, 6-keto-prostaglandin $F 1 \alpha$, prostaglandin $F 1 \alpha$, and TXB2<0.01\%.

Urine creatinine concentration was determined using Detect $\mathrm{X}^{\mathrm{TM}}$ competitive enzyme immunoassay (Arbor Assays LLC, Ann Arbor, MI) with plates read at spectrophotometer settings at $490 \mathrm{~nm}$ as per the manufacturer's instructions. This assay has been validated for use with dog urine by the manufacturer and the intra-assay and inter-assay coefficients of variation were reported as $3.0 \%$ and $3.9 \%$ or less, respectively. Results for ISO are reported as $\mathrm{pg} / \mathrm{mL}$ divided by creatinine in $\mathrm{mg} / \mathrm{dL}$. Results for TXB2, PGF1 $\alpha$, prostaglandin E2 metabolites, 
and PGF $2 \alpha$ are reported as $\mathrm{pg} / \mathrm{mL}$ divided by creatinine in $\mathrm{mg} / \mathrm{dL}$.

\section{Statistical analyses}

Values are reported as the mean \pm standard deviation and median, range. A Nemenyi-Damico-Wolfe-Dunn Test was used to detect differences between sample times (baseline prior to exercise, immediately post-exercise, and 4-hours post-exercise) and skill levels of the dogs (novice, intermediate, masters) [18]. Significance was set at $\mathrm{P}<0.05$.

\section{Results}

\section{Skill groups}

Fifteen dogs were enrolled in the study. Masters dogs were an average of $6.2 \pm 2.0$ (median 7.0, range 4-8) years of age $(n=5)$. Novice dogs were $2.8 \pm 1.5$, median 2.5 , range $1-5$ years, $n=6$ ) which was slightly younger than intermediate level dogs $(7.0 \pm 2.3$ years, median 7.0 and range $5-9, n=4 ; \mathrm{P}>0.05)$. Seven of the 15 dogs were spayed females and the rest were neutered males. The masters level dogs had spent $4.0 \pm 1.4$ years performing agility (median 5.0 and range 2-5 years), the intermediate level dogs $3.0 \pm 0.8$ (median 3.0, range 2-4) years and the novice level dogs $1.2 \pm 1.1$ (median 0.8, range 0.2-3) years. The height of the jumps was not different between the skill groups (novice $16 \pm 4.4(16,12-20)$ inches, intermediate $16 \pm 0.0$ (all dogs 16 inches), masters $15.2 \pm$ $5.2(16,8-20)$ inches, $P>0.05)$. The breeds in the masters level group included two mixed breeds, a Border Collie, a Jack Russell Terrier, and a Pembroke Welsh Corgi. The intermediate level dogs contained a Golden Retriever, a Belgian Terrier, an Australian Shepherd, and a Standard Poodle. The novice level group contained a Golden Retriever, a Boxer, a Standard Poodle, a Miniature Poodle, a Welsh Terrier, and a Queensland Heeler. Six of the 15 dogs were administered glucosamine and chondroitin sulfate supplements daily. Two dogs were intermittently treated with antibiotics and antihistamines for owner reported skin allergies. Two other dogs had been previously diagnosed as hypothyroid and were receiving thyroid supplementation that upon testing resulting in serum T4 concentrations within the reference range for the lab. The frequency with which each group trained their dogs was not different between the groups and on average the masters level dogs spent $4.2 \pm 1.8$ (median 4.0, range 2-6) hours per week in training while the intermediate level dogs spent $4.0 \pm 1.4(3.5,3-6)$ hours and the novice level dogs $3.0 \pm$ 0.9 (3.0, 2-4) hours per week in training, respectively, $\mathrm{P}>0.01$.

The time taken to complete the course was significantly different between the skill groups (two-way ANOVA, $\mathrm{P}=0.0092$ ) and for each run (run 1 , run 2 or run3, $\mathrm{P}=0.01$ ). The masters level dogs took the least time to complete their runs [run 1, $29.5 \pm 9.5$ (median 25.4, range 23.8-46.3), run $2,29.4 \pm 10.0$ (25.3, 22.9-44.2), run 3, $29.2 \pm 8.4(25.8,23.6-43.8)$ seconds] and had the least mistakes (such as missed jumps) compared to the intermediate level [run 1, $38.8 \pm 9.7$ (median 39.8, range 27.9-47.8), run 2, $41.3 \pm 18.2$ (32.9, 30.8-68.4), run 3, $49.8 \pm$ 39.5 (30.4, 29.4-109) seconds] or novice level dogs (run 1 $33.6 \pm 7.5(31.5,26.2-45.0)$, run $2,33.2 \pm 4.8$ (32.4, 28.2-40.5), run 3, $34.3 \pm 1.0$ (34.4, 30.7-37.5) seconds).

\section{Physical variables}

Body temperature increased significantly immediately following the exercise test, but returned to pre-exercise values by 4 -hours after the test (pre-exercise 101.4 \pm 0.8 , median 101.3 , range $100.2-102.7^{\circ} \mathrm{F}$, immediately postexercise $102.4 \pm 1.3$, median $102.1,100.3-103.6^{\circ} \mathrm{F}$, and 4-hours post $100.4 \pm 1.0$, median 100.8 , range $98.1-101.7^{\circ} \mathrm{F}$, $\mathrm{P}<0.0001)$. Body temperature was unaffected by skill level $(\mathrm{P}=0.3)$. Heart rate was significantly higher immediately following the exercise test and decreased significantly 4 hours after the test compared to pre-test and immediate post-test values for all groups of dogs (pre-exercise $104 \pm$ 21.5 , median 96 , range $84-148$, immediately post-exercise $136.5 \pm 19.7$, median 130, range 110-180, 4-hours post $83.6 \pm 25.9$, median 74 , range $52-133$ beats per minute, $\mathrm{P}<0.0001)$. As with body temperature, heart rate was not affected by the dogs' skill level $(\mathrm{P}=0.07)$.

\section{Hematological profile}

Results of the hematology analysis are reported in Table 1. Red blood cell count (RBC), hematocrit (HCT), hemoglobin, and serum albumin increased immediately following the agility exercise $(\mathrm{P}<0.05)$. Albumin decreased to values similar to pre-exercise values by 4 -hours following the exercise test, however, RBC, HCT and hemoglobin were decreased at 4-hours compared to preexercise values. RBC, HCT, hemoglobin, and albumin were different in different skill groups; they increased the greatest in the masters group followed by intermediates and the least in the novice dogs immediately after exercise $(\mathrm{P}<0.05)$. None of the other hematological parameters examined (white blood cell count, total plasma protein, platelet count, mean corpuscular volume, mean corpuscular hemoglobin concentration, neutrophil count, lymphocyte count, monocyte count, eosinophil count, basophil count) were affected by time or skill level of the dogs, $\mathrm{P}>0.05$.

\section{Biochemistry profile}

Biochemistry parameters are reported for each skill group and time point in Table 2. Serum creatinine and total plasma protein were not affected by the exercise test or skill level $(P>0.05)$. Lactate increased significantly immediately after the exercise test $(\mathrm{P}<0.001)$ and differed between skill groups, Table $2 \quad(\mathrm{P}<0.001)$. Lactate increased most in the masters skill group, then the 
Table 1 Hematological profile results

\begin{tabular}{|c|c|c|c|c|c|c|c|c|c|c|}
\hline & \multicolumn{3}{|c|}{ Novice } & \multicolumn{3}{|c|}{ Intermediate } & \multicolumn{3}{|c|}{ Masters } & \multirow[t]{2}{*}{ Reference range } \\
\hline & Pre & Post & 4 hr Post & Pre & Post & 4 hr Post & Pre & Post & 4 hr Post & \\
\hline \multirow[t]{2}{*}{ RBC $\S\left(x 10^{6} / \mu \mathrm{L}\right)$} & $7.0 \pm 0.6$ & $7.3 \pm 0.2$ & $6.8 \pm 0.6$ & $6.3 \pm 0.9$ & $7.0 \pm 1.0$ & $6.1 \pm 0.7$ & $6.2 \pm 0.3$ & $7.1 \pm 0.4$ & $6.1 \pm 0.4$ & $5.5-8.5$ \\
\hline & $7.1,6-7.6$ & $7.3,6.9-7.5$ & $6.7,6.2-7.6$ & $6.6,5-6.9$ & $6.9,5.8-8.2$ & $6.2,5.1-6.8$ & $6.4,5.8-6.5$ & $6.9,6.6-7.7$ & $6.2,5.6-6.5$ & \\
\hline \multirow[t]{2}{*}{ НCT (\%)§ } & $47.3 \pm 3.7$ & $49.1 \pm 1.3$ & $45.3 \pm 2.8$ & $42.1 \pm 5.4$ & $47.4 \pm 6.2$ & $40.9 \pm 5.0$ & $43.5 \pm 3.1$ & $49.7 \pm 4.0$ & $42.7 \pm 3.1$ & $37-55$ \\
\hline & $48.1,41.4-52.1$ & $47-50.9$ & $45.3,42-49.5$ & $43.9,34.2-46.3$ & $46.4,41-55.8$ & $41.4,34.5-46.3$ & $44.3,38.7-47.1$ & $50.2,43.8-54.4$ & $38.7-46.6$ & \\
\hline \multirow[t]{2}{*}{$\mathrm{Hgb}(\mathrm{g} / \mathrm{dL}) \S$} & $16.9 \pm 1.3$ & $17.7 \pm 0.3$ & $16.4 \pm 1.1$ & $15.4 \pm 1.6$ & $16.8 \pm 2.4$ & $14.7 \pm 1.8$ & $15.9 \pm 0.9$ & $18 \pm 1.2$ & $15.4 \pm 1.0$ & $12-18$ \\
\hline & $17.2,14.7-18.4$ & $17.7,17.2-18.1$ & $16.3,15.3-17.3$ & $15.8,13-16.8$ & $16.4,14.4-20$ & $14.8,12.4-16.7$ & $16,14.5-16.8$ & $17.9,16.7-19.8$ & $15.3,14.1-16.7$ & \\
\hline \multirow[t]{2}{*}{ 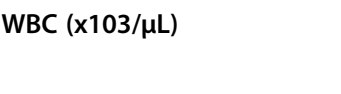 } & $8.9 \pm 2.9$ & $9.4 \pm 3.2$ & $9.6 \pm 3.1$ & $6.7 \pm 1.5$ & $8.2 \pm 1.3$ & $7.2 \pm 1.4$ & $6.5 \pm 1.3$ & $7.5 \pm 1.8$ & $7.1 \pm 1.5$ & $6-17$ \\
\hline & $8.0,6.5-14.1$ & $8.3,6.1-15$ & $9.2,6.7-15.2$ & $6.7,5.1-8.3$ & $8.7,6.3-9.2$ & $7.3,5.5-8.5$ & $6.2,5.2-8.5$ & $6.4,6.2-10.4$ & $7.5,5.1-8.6$ & \\
\hline \multirow[t]{2}{*}{ Platelets $(x 1000 / \mu \mathrm{L})$} & $269 \pm 76$ & $263 \pm 87$ & $236 \pm 68$ & $253 \pm 27$ & $274 \pm 41$ & $236 \pm 31$ & $286 \pm 46$ & $319 \pm 58$ & $242 \pm 85$ & $200-900$ \\
\hline & $272,141-347$ & $267,127-354$ & $241,156-314$ & $251,226-284$ & $272,229-324$ & $238,200-269$ & $293,211-334$ & 233-391 & $279,115-333$ & \\
\hline \multirow[t]{2}{*}{ Albumin $\S(g / d L)$} & $3.8 \pm 0.2$ & $3.9 \pm 0.1$ & $3.9 \pm 0.2$ & $3.7 \pm 0.1$ & $3.9 \pm 0.1$ & $3.7 \pm 0.1$ & $3.6 \pm 0.1$ & $3.8 \pm 0.1$ & $3.6 \pm 0.1$ & $2.3-4.0$ \\
\hline & $3.8,3.6-4.0$ & $3.9,3.7-4.1$ & $3.8,3.6-4.1$ & $3.7,3.6-3.8$ & $3.9,3.8-4.1$ & $3.7,3.6-3.9$ & $3.6,3.6-3.8$ & $3.8,3.6-4.0$ & $3.7,3.5-3.8$ & \\
\hline \multirow[t]{2}{*}{ Total Plasma Protein ( $\mathrm{g} / \mathrm{dL})$} & $6.1 \pm 0.4$ & $6.2 \pm 0.3$ & $6.1 \pm 0.5$ & $6.5 \pm 0.56 .5$ & $6.5 \pm 0.4$ & $6.1 \pm 0.4$ & $6.2 \pm 0.3$ & $6.5 \pm 0.2$ & $6.0 \pm 0.3$ & $5.4-7.6$ \\
\hline & $6.1,5.6-6.8$ & $6.2,5.8-6.6$ & $6.0,5.5-6.8$ & $5.8-7.0$ & $6.6,6.1-6.8$ & $6.1,5.6-6.5$ & $6.0,6.0-6.6$ & $6.5,6.1=6.7$ & $6.1,5.7-6.4$ & \\
\hline
\end{tabular}

Values are mean \pm standard deviation, median and range; differences were detected using the Memenyi-Damico-Wolfe-Dunn Test $\$$ Parameter significantly affected by the exercise test (i.e. pre or post exercise test, $\mathrm{WBC}=$ white blood cell count, $\mu \mathrm{L}=$ microliter, $\mathrm{g} / \mathrm{dL}=$ grams per deciliter. 
Table 2 Serum biochemistry profile results

\begin{tabular}{|c|c|c|c|c|c|c|c|c|c|c|}
\hline & \multicolumn{3}{|c|}{ Novice } & \multicolumn{3}{|c|}{ Intermediate } & \multicolumn{3}{|c|}{ Masters } & \multirow{2}{*}{$\begin{array}{c}\text { Reference } \\
\text { range }\end{array}$} \\
\hline & Pre & Post & 4 hr Post & Pre & Post & 4 hr Post & Pre & Post & 4 hr Post & \\
\hline $\begin{array}{l}\text { Lactate } \S \\
(\mathrm{mmol} / \mathrm{L})\end{array}$ & $\begin{array}{r}1.04 \pm 0.35 \\
1.1,0.58-1.47\end{array}$ & $\begin{array}{c}1.28 \pm 0.55 ; 1.22 \\
0.82-2.34\end{array}$ & $\begin{array}{c}0.64 \pm 0.20 \\
0.6,0.40-0.97\end{array}$ & $\begin{array}{l}2.34 \pm 1.66 \\
1.6,1.4-4.82\end{array}$ & $\begin{array}{c}7.39 \pm 5.43 \\
6.78,1.42-14.60\end{array}$ & $\begin{array}{c}2.10 \pm 1.97 \\
1.55,0.42-4.86\end{array}$ & $\begin{array}{c}1.10 \pm 0.41 \\
1.0,0.64-1.69\end{array}$ & $\begin{array}{c}3.93 \pm 2.53 \\
3.56,0.87-7.89\end{array}$ & $\begin{array}{c}0.69 \pm 0.27 \\
0.52,0.49-1.05\end{array}$ & $<4$ \\
\hline $\begin{array}{l}\text { Creatinine } \\
\text { (mg/dL) }\end{array}$ & $\begin{array}{c}1.0 \pm 0.3 \\
1.0,0.7-1.3\end{array}$ & $\begin{array}{c}0.9 \pm 0.3 \\
1.0,0.6-1.2\end{array}$ & $\begin{array}{c}0.8 \pm 0.3 \\
0.9,0.5-1.1\end{array}$ & $\begin{array}{c}1.0 \pm 0.3 \\
1.1,0.5-1.2\end{array}$ & $\begin{array}{l}1.1 \pm 0.4 \\
1.2, .5-1.4\end{array}$ & $\begin{array}{c}0.9 \pm 0.3 \\
0.9,0.5-1.1\end{array}$ & $\begin{array}{c}0.9 \pm 0.4 ; 0.9 \\
0.6-1.5\end{array}$ & $\begin{array}{c}1.0 \pm 0.4 \\
1.0,0.7-1.6\end{array}$ & $\begin{array}{c}0.8 \pm 0.3 \\
0.8,0.7-1.4\end{array}$ & $1-2$ \\
\hline BUN (mg/dL) & $\begin{array}{c}\text { 18. } \pm 3 \\
19,13-21\end{array}$ & $\begin{array}{c}17 \pm 3 \\
17,12-21\end{array}$ & $\begin{array}{c}16 \pm 4 \\
16,10-22\end{array}$ & $\begin{array}{c}19 \pm 4 \\
19,14-24\end{array}$ & $\begin{array}{c}19 \pm 4 \\
20,14-23\end{array}$ & $\begin{array}{c}17 \pm 4 \\
18,12-21\end{array}$ & $\begin{array}{c}17 \pm 5 \\
17,10-23\end{array}$ & $\begin{array}{c}18 \pm 5 \\
17,12-23\end{array}$ & $\begin{array}{c}18 \pm 2 \\
18,16-21\end{array}$ & $10-30$ \\
\hline $\begin{array}{l}\text { Creatinine } \\
\text { Kinase (IU/L) }\end{array}$ & $\begin{array}{c}138 \pm 59 \\
123,88-248\end{array}$ & $\begin{array}{c}100 \pm 23 \\
103,62-130\end{array}$ & $\begin{array}{c}225 \pm 158 \\
140,102-448\end{array}$ & $\begin{array}{l}110 \pm 45 \\
98,72-173\end{array}$ & $\begin{array}{r}142 \pm 105 \\
114,63-281\end{array}$ & $\begin{array}{l}150 \pm 134 \\
91,67-349\end{array}$ & $\begin{array}{c}108 \pm 52 \\
108,48-189\end{array}$ & $\begin{array}{c}136 \pm 64 \\
131,48-218\end{array}$ & $\begin{array}{c}116 \pm 49 \\
107,50-180\end{array}$ & $50-300$ \\
\hline Calcium (mmol/L) & $\begin{array}{c}10.5 \pm 0.2 \\
10.6,10.2-10.8\end{array}$ & $\begin{array}{c}10.4 \pm 0.4 \\
10.49 .6-10.6\end{array}$ & $\begin{array}{c}10.5 \pm 0.1 \\
10.5,10.4-10.7\end{array}$ & $\begin{array}{c}10.3 \pm 0.4 \\
10.4,9.8-10.6\end{array}$ & $\begin{array}{c}10.6 \pm 0.2 \\
10.6,10.3-10.8\end{array}$ & $\begin{array}{c}10.2 \pm 0.1 \\
10.2,10.0-10.3\end{array}$ & $\begin{array}{c}10.1 \pm 0.1 \\
10.1,9.9=10.3\end{array}$ & $\begin{array}{c}10.3 \pm 0.2 \\
10.3,10.1-10.5\end{array}$ & $\begin{array}{c}10.2 \pm 0.4 \\
10.3,9.6-10.7\end{array}$ & $8-12$ \\
\hline $\begin{array}{l}\text { Phosphorus § } \\
\text { (mmol/L) }\end{array}$ & $\begin{array}{c}3.9 \pm 0.5 \\
3.9,3.3-4.7\end{array}$ & $\begin{array}{l}4.2 \pm 0.6 \\
4.3,3.1-5\end{array}$ & $\begin{array}{c}4.7 \pm 0.2 \\
4.7,4.4-4.9\end{array}$ & $\begin{array}{c}3.3 \pm 0.2 \\
3.3,3.1-3.6\end{array}$ & $\begin{array}{l}3.7 \pm 0.3 \\
3.8,3.3-4\end{array}$ & $\begin{array}{r}4.2 \pm 0.5 \\
4.2,3.7-4.7\end{array}$ & $\begin{array}{r}3.9 \pm 0.7 \\
3.8,2.9-4.8\end{array}$ & $\begin{array}{l}3.9 \pm 0.7 \\
3.7,3.3-4\end{array}$ & $\begin{array}{l}4.6 \pm 0.6 \\
4.3,4-5.4\end{array}$ & $3-7$ \\
\hline Sodium (mEq/L) & $\begin{array}{c}146.8 \pm 1.5146 .9 \\
144.4-149.2\end{array}$ & $\begin{array}{c}148 \pm 1 \\
149,147-149\end{array}$ & $\begin{array}{c}148 \pm 3 \\
148,146-154\end{array}$ & $\begin{array}{c}146.7 \pm 3.57146 .6 \\
142.5-151.2\end{array}$ & $\begin{array}{c}151 \pm 1 \\
151,149-152\end{array}$ & $\begin{array}{c}146 \pm 5 \\
147,140-150\end{array}$ & $\begin{array}{c}145.2 .6 \pm 2.1 \\
146.1,141.7-146.7\end{array}$ & $\begin{array}{c}149 \pm 1.6 \\
149,146-150\end{array}$ & $\begin{array}{c}147 \pm 2 \\
148,145-149\end{array}$ & $140-158$ \\
\hline $\begin{array}{l}\text { Potassium } \\
(\mathrm{mEq} / \mathrm{L})\end{array}$ & $\begin{array}{c}4.2 \pm 0.2 \\
4.2,3.9-4.3\end{array}$ & $\begin{array}{l}4.4 \pm 0.2 \\
4.5,4-4.5\end{array}$ & $\begin{array}{c}4.1 \pm 0.8 \\
4.3,2.5-4.8\end{array}$ & $\begin{array}{c}4.1 \pm 0.1 \\
4.1,3.9-4.2\end{array}$ & $\begin{array}{l}4.4 \pm 0.5 \\
4.3,4-5.1\end{array}$ & $\begin{array}{c}4.1 \pm 0.4 \\
4.2,3.6-4.4\end{array}$ & $\begin{array}{c}4.1 \pm 0.2 \\
4.1,3.9-4.4\end{array}$ & $\begin{array}{c}4.3 \pm 0.4 \\
4.3,3.8-4.9\end{array}$ & $\begin{array}{c}4.1 \pm 0.4 \\
4.3,3.5-4.4\end{array}$ & $4.0-5.7$ \\
\hline Chloride (mEq/L) & $\begin{array}{c}114 \pm 2 \\
114,110-115\end{array}$ & $\begin{array}{c}114 \pm 2 \\
115,111-116\end{array}$ & $\begin{array}{c}113 \pm 2 \\
113,111-117\end{array}$ & $\begin{array}{c}114 \pm 5 \\
116 ; 106-118\end{array}$ & $\begin{array}{c}113 \pm 6 \\
115,105-119\end{array}$ & $\begin{array}{c}111 \pm 6 \\
113,103-117\end{array}$ & $\begin{array}{c}114 \pm 3 \\
114,111-118\end{array}$ & $\begin{array}{c}113 \pm 2 \\
113,110-114\end{array}$ & $\begin{array}{c}114 \pm 3 \\
115,110-117\end{array}$ & $100-115$ \\
\hline ALT (IU/L) & $\begin{array}{c}50 \pm 31 \\
38,27-111\end{array}$ & $\begin{array}{c}50 \pm 30 \\
40,28-110\end{array}$ & $\begin{array}{c}52 \pm 33 \\
40,29-119\end{array}$ & $\begin{array}{c}41 \pm 7 \\
42,32-46\end{array}$ & $\begin{array}{c}47 \pm 9 \\
47,36-57\end{array}$ & $\begin{array}{c}45 \pm 8 \\
47,33-52\end{array}$ & $\begin{array}{c}70 \pm 72 \\
31 ; 27-194\end{array}$ & $\begin{array}{l}77.0 \pm 76 \\
36,31-209\end{array}$ & $\begin{array}{c}73 \pm 71 \\
37,30-197\end{array}$ & $5-65$ \\
\hline ALP (IU/L) & $\begin{array}{c}47 \pm 28 \\
35,21-87\end{array}$ & $\begin{array}{l}47 \pm 29 \\
32,22-89\end{array}$ & $\begin{array}{l}43 \pm 28 \\
29,20-82\end{array}$ & $\begin{array}{c}53 \pm 32 \\
47,32-46\end{array}$ & $\begin{array}{l}58 \pm 29 \\
55,32-91\end{array}$ & $\begin{array}{l}54 \pm 30 \\
51,27-87\end{array}$ & $\begin{array}{r}30 \pm 10 \\
28,20-42\end{array}$ & $\begin{array}{c}30 \pm 11 \\
28,21-46\end{array}$ & $\begin{array}{c}30 \pm 11 \\
24,19-44\end{array}$ & $10-84$ \\
\hline Glucose (mg/dl) & $\begin{array}{c}90 \pm 14 \\
90,68-107\end{array}$ & $\begin{array}{c}94 \pm 14 \\
95,76-114\end{array}$ & $\begin{array}{c}98 \pm 11 \\
97,84-116\end{array}$ & $\begin{array}{c}89 \pm 18 \\
89,69-108\end{array}$ & $\begin{array}{c}104 \pm 26 \\
103,74-136\end{array}$ & $\begin{array}{c}95 \pm 7 \\
95,85-103\end{array}$ & $\begin{array}{c}99 \pm 5 \\
98,93-105\end{array}$ & $\begin{array}{c}103 \pm 7 \\
100,97-114\end{array}$ & $\begin{array}{c}99 \pm 5 \\
97,95-107\end{array}$ & $65-130$ \\
\hline
\end{tabular}

sample was taken, $\mathrm{P}<0.01$; however there were no differences between the skill groups. For lactate, groups with the same superscript are significantly different between skill groups and the time the sample was taken
sactions (i.e. pre and post exercise test, $\mathrm{P}<0.01$ ). Pre $=$ pre-exercise, Post $=$ post-exercise, $4 \mathrm{hr}$ post $=4$ hours following exercise, $\mathrm{BUN}=$ blood urea nitrogen, $\mathrm{ALT}=$ alanine aminotransferase, $\mathrm{ALP}=$ alkaline phosphatase, $\mathrm{mmol} / \mathrm{L}=$ millimoles per liter, $\mathrm{mg} / \mathrm{L}=$ milligrams per deciliter, $\mathrm{IU} / \mathrm{L}=$ international units per liter, $\mathrm{mEq} / \mathrm{L}=$ milliequivalents per liter. 
intermediate group, and it changed little in the novice dogs following agility exercise. Phosphorus increased in dogs immediately after the exercise test and continued to rise 4-hours following the test, with the greatest increase in novice and intermediate dogs and least change in the masters group $(\mathrm{P}<0.05)$. Creatinine kinase activity increased slightly but not significantly following exercise in all groups (pre-exercise $124.0 \pm 12.9$, immediately post-exercise $139.1 \pm 22.2$, 4 hours post-exercise $190.8 \pm$ 37.7 U/L). Creatinine kinase was affected by skill level of the dogs with the novice group having the highest values $(\mathrm{P}<0.05$, Table 2$)$. Blood urea nitrogen, calcium, sodium, potassium and chloride did not significantly change over time or between dog skill levels, $\mathrm{P}>0.05$.

\section{Oxidant stress}

Urinary creatinine excretion was $275.4 \pm 118.0$ (median 262.9, range 41-593.5 mg/dL) pre-exercise, $205.7 \pm 116.7$ (median 213.1, range 29.9-446.8) $\mathrm{mg} / \mathrm{dL}$ and at 4 hours post-exercise $223.8 \pm 103.8$ (median 222.1, range 36.7418.2) $\mathrm{mg} / \mathrm{dL}$. While urinary creatinine was not affected by the exercise test, it was significantly less in the intermediate group of dogs compared to the other groups $(\mathrm{P}<0.01)$. Urinary excretion of ISO increased following agility exercise and remained elevated 4 hours after the exercise test [pre-exercise $14.50 \pm 5.23$ (median 14.62, range 2.48-20.70), post-exercise $20.31 \pm 12.95$ (median 16.20, range 9.46-60.70) and 4-hours post-exercise $19.00 \pm$ 4.95 (median 18.44, range 12.05-26.75) pg/mL per $\mathrm{mg} / \mathrm{dL}$ creatinine, $\mathrm{P}>0.05]$, however, this mild increase was not statistically significant.

\section{Urinary eicosanoid excretion}

Urinary prostaglandin $\mathrm{E}_{2}$ metabolites (13,14-dihydro-15keto-prostaglandin $\mathrm{A}_{2}$ and 13,14-dihydro-15-keto-prostaglandin $\mathrm{E}_{2}$ ) and 13,14-dihydro-15-keto prostaglandin $\mathrm{F}_{2 \alpha}$ were not affected by the agility exercise at any time point (Table, two-way ANOVA, P>0.05). Prostacyclin (6-keto prostaglandin $F_{1 \alpha}$ ) excretion increased only in masters dogs compared to intermediate dogs at 4 hours following the exercise test (two-way ANOVA, Bonferroni post-test, $\mathrm{P}=0.04$ ). Urinary TBX2 more than tripled by 4 hours post agility exercise (pre 4.56 , post $11.76,4$ hour post 14.91 $\mathrm{pg} / \mathrm{ml} / \mathrm{mg} / \mathrm{dl}$ creatinine, $\mathrm{P}=0.005$, one-way ANOVA, GraphPad Prism 5.0a, La Jolla, CA) with a significant increase in masters dogs (pre $4.55 \pm 0.62$, post $8.35 \pm 1.06$, 4 hour post $15.27 \pm 2.76, \mathrm{P}=0.0097)$ but not novice (pre $4.50 \pm 0.70$, post $9.89 \pm 2.67,4$ hour post $7.21 \pm 2.01$, $\mathrm{P}=0.19$ ) or intermediate dogs (pre $6.68 \pm 2.60$, post $11.72 \pm$ 4.97, 4 hour post $10.53 \pm 5.22, \mathrm{P}=0.08$, one-way ANOVA), Figure 1. Urinary 11-TXB2 significantly increased over time $(\mathrm{P}=0.035)$ but did not differ between the skill groups: novice pre $19.07 \pm 1.29$, post $25.80 \pm 1.43$, 4 hour post $25.37 \pm 1.82$ ); intermediate dogs pre $5.49 \pm 1.47$, post

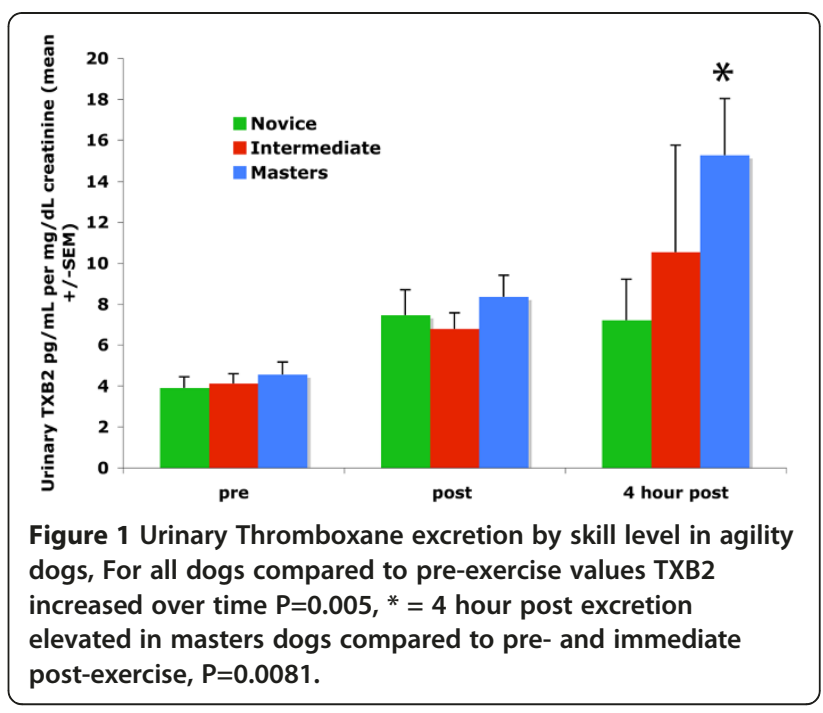

$10.60 \pm 0.74,4$ hour post $10.99 \pm 0.66$; masters pre $3.79 \pm 1.69$, post $15.15 \pm 2.22$, 4 hour post $13.65 \pm 1.52$ (P>0.05, two-way ANOVA).

\section{Discussion and conclusions}

The skill levels of the dogs reported in this study was determined by the rules set out by the USDAA and may have seemed arbitrary. The groupings proved accurate in these dogs, however, since each successive skill group was significantly faster than the novice group and the group level below it. The labeling of these groups as novice, intermediate and masters could have been labeled instead by their time through the simulated exercise test, however, this would have been less applicable to dogs in actual agility competition.

Agility exercise, as performed by dogs in this study, is of sufficient intensity to induce mild oxidant stress and lipid peroxidation based upon the slightly increased urinary excretion of ISO following the exercise test. The intensity of muscle contraction associated with agility exercise increases production of reactive oxygen species due to increased oxygen consumption, resulting in elevated superoxide anion production in skeletal muscle mitochondria [19]. All dogs in this study had similar increases in ISO regardless of skill level and speed through the course, indicating that agility exercise routinely stimulates intense muscle contraction and results in mild lipid peroxidation. It is unlikely, given the results reported here, that the mild oxidant stress encountered in a single agility exercise session has a significant effect on physiologic function and performance. Previous reports have documented oxidative stress in dogs exercising for 20 minutes (hunting dogs) and many hours (sled dogs), however, this is the first report, to the authors' knowledge, of the effect of very short duration, intense exercise on lipid peroxidation and oxidation in 
the dog $[13,20,21]$. Many agility dogs participate in more than one trial in a single day or weekend. In humans, intense exercise elevates urinary ISO excretion for 24 hours, and following a soccer game, humans experience oxidant stress and reduced performance for up to 72 hours [12,22]. Muscle fatigue due to oxidant stress may be a contributing factor to joint injuries, at least in humans [23,24]. Whether oxidant stress plays a role in injury to dogs during agility competition with repeated runs through a course in a single day remains unknown, however determining the level of oxidant stress that develops over time and the length of time before isoprostane production returns to pre-exercise levels in actual agility competition (rather than simulated as reported here) may be warranted based upon the results of this study and the high incidence of injury following participation in this activity [2]. Collection of urine at more precise time points following the agility exercise may have altered the results in our study by reducing variability between the subjects. Future research determining other markers of oxidant stress at exact time points prior to and following agility exercise is indicated by the results of this study.

A significant limitation of this study is the use of an EIA for determination of $F_{2}$ isoprostanes in the urine of dogs. Analysis of the methods used to determine urinary $\mathrm{F}_{2}$ isoprostane excretion have found that EIA results do not correlate with gas chromatography negative ion chemical ionization-mass spectroscopy (GC/NICI-MS, considered the gold standard) in dogs [25]. The results of this study cannot be compared to other studies, however, mildly increased lipid peroxidation following the intense, short-duration exercise of agility competition occurred in the dogs reported here and was not affected by the speed or training of the dogs. Further research, preferably using GC/NICI-MS for urinary $F_{2}$ isoprostane determination, is required to determine the extent of oxidant stress induced by this type of exercise in relation to long duration exercise in sled dogs and to disease states including lung injury [26], neurologic disease [16] and heart disease [27]. Future research is warranted to determine the effect of agility exercise on other parameters of oxidant stress including glutathione, glutathione-peroxidase, catalase, superoxide dismutase.

Another limitation of this study is the lack of data regarding antioxidant status in these dogs. Antioxidants are either supplements or endogenous mechanisms of consumption or removal of reactive oxygen species and include superoxide dismutase, catalase, glutathioperoxidase, glutathione reductase, vitamin $\mathrm{E}$, vitamin $\mathrm{C}$, and ubiquinone [28]. Hunting dogs exercised for 20 minutes to 4 hours duration decrease their biological antioxidant potential following the exercise but the return of this potential is within one hour [29]. Determination of antioxidant status may be warranted in future studies of dogs participating in agility exercise.

The plasma lactate increased markedly in the dogs following the exercise test. The changes in plasma lactate were inversely proportional to the speed through the course (and proportional to increasing skill level). Plasma lactate concentration at a specific speed or level of intensity is strongly correlated to physical fitness in horses as well as human athletes [30,31]. In the current study, all three skill groups had normal plasma lactate concentrations by 4 hours after exercise, indicating that recovery from lactic acidosis in agility dogs occurs quickly, similar to other athletic events, and before recovery from oxidative stress [32].

In Greyhounds following high intensity sprint exercise, plasma lactate increased to $29.3 \mathrm{meq} / \mathrm{L}$ or more, and similar to the dogs reported here, returns to baseline in less than 4 hours following the exercise [32-35]. During field trial competition, Labrador retrievers also increase plasma lactate concentrations similar to the masters level dogs in this study [36-38]. Increased plasma lactate was most likely the result of anaerobic metabolism in contracting muscles during the sprint exercise [32]. Studies of the Greyhound have indicated that this breed has higher concentrations of type II fast twitch muscle fibers [39]. These fibers have the greatest glycolytic/ anaerobic capacity and therefore are capable of producing large amounts of lactate during high intensity exercise [40]. The relative amount of muscle fiber types present in the dogs in this study is unknown, however, a higher percentage of type II fibers may have resulted in greater increases in plasma lactate following the exercise test and significantly shorter times through the agility course. Elite sprinters in men and horses have higher proportions of type II fibers than their slower counterparts and plasma lactate has been shown to correlate with racing speed in thoroughbred horses and female cyclists [41-44].

Lactic acidosis and oxidant stress may be causes of muscle fatigue [45]. The mechanism by which reactive oxygen species cause muscle fatigue during intense exercise is not fully understood, however, supplementation with the antioxidant, $\mathrm{N}$-acetylcysteine alleviates muscle fatigue in humans and animals [46-49]. Muscle fatigue due to oxidant stress may be a contributing factor to joint injuries, at least in humans [23,24]. Increasing lactate production by high intensity-contracting muscle will result in decreased ionized calcium release from the sarcolemma and contribute to muscle fatigue [50]. Muscle fatigue has been linked to increased bone strain in dogs experimentally, and may contribute to the development of stress fractures [51,52]. Further research is warranted to determine the muscle fiber types of elite agility competitors and whether, in competition, plasma lactate correlates with injury rates in these dogs. 
Platelets increased immediately following the agility exercise but by 4 hours later had returned to values similar to pre-exercise; and at all time-points, values were within reference range for the laboratory (200-900 $\times 1000 / \mu \mathrm{l})$. In humans, increased platelet count occurs with intense exercise and are thought to be mobilized from the spleen, bone marrow and lungs, but they may also increase due to hemoconcentration from respiratory water loss as a result of panting [53-55]. The small reduction in platelet counts following exercise may be due to plasma volume expansion that occurs during recovery or reduced catecholamine-induced platelet release $[53,55]$.

Similar to previous reports in agility dogs, increases in red blood cell count, hemoglobin and hematocrit occurred in all groups immediately following exercise $[3,4]$. Increases in these variables without a rise in total plasma protein is consistent with splenic contraction with similar findings reported in Greyhounds after racing [56]. RBC count, HCT, and hemoglobin concentrations were decreased at 4-hours following the agility exercise test. In humans, during the hours following exercise, there is an increase in plasma volume termed "autohemodilution" resulting in decreased concentrations of RBC's, HCT and hemoglobin [57-59]. This phenomenon may explain the decreased red blood cell parameters in the dogs reported here at 4-hours following exercise without alteration in protein or albumin concentrations as fluid from the interstitial space moves into the vascular space. To the authors' knowledge, this is the first report indicating "autohemodilution" in dogs and further research is needed. Plasma albumin concentration increased immediately after exercise $(\mathrm{P}<0.01)$ but remained within reference range and returned to pre-exercise values within 4 hours, Table 1 . No other alterations in hematological or biochemical parameters were found.

Increased TXB2 production without concomitant increase in prostaglandin $E_{2}$ or PGF1 $\alpha$ as reported here in agility dogs has also been reported in horses following treadmill exercise [60]. In endurance exercise, horses may also increase 6-keto-prostaglandin $\mathrm{F}_{1 \alpha}$ production, however this did not occur in the dogs of this study where the duration of exercise lasted less than 20 minutes [15]. The dogs with the greatest skill level (and fastest run time) had a significantly greater increase in TXB2 and this association has also been reported in horses [15]. High intensity exercise reportedly stimulates increased TXB2 production whereas submaximal exercise induces increases in PGF1 $\alpha$ rather than TXB2 in humans [61]. Urinary 11-TXB2 has been determined to be a reflection of in vivo platelet activation in humans [62]. TXB2 in urine includes renal production of thromboxane $\mathrm{A}_{2}$ and may not reflect systemic circulation or platelet production of the unstable prostaglandin, thromboxane $A_{2}$ [63]. In this study both TXB2 and 11-TXB2 increased following exercise and remained increased 4 hours post exercise indicating systemic increases in thromboxane A2 occurred in the dogs. Interestingly, only TXB2 was significantly increased when skill levels were examined and not 11-TXB2 which may indicate greater renal production of thromboxane A2 only in the fastest dogs. Potential sources of TXB2 may include endothelial cells activated by shear stress or catecholamine activated platelets, however the source was not determined in this study. Future research to determine why improved performance is associated with increased TXB2 in the urine is warranted.

The dogs participating in the exercise test were not controlled for breed, age, sex, or nutritional status. Restriction of these variables was not enforced since a very wide variety of dogs participate in agility sports and many of them go on to injure themselves in this exercise; therefore, we sought to ascertain the degree of physiological alteration that occurs in a variety of agility dogs. Future research may limit the participants by breed etc. and investigate dogs during actual agility competition rather than a simulated test.

Agility competition in dogs has become a popular sport with a high incidence of injury [2]. Development of methods to reduce increases in urinary TXB2 and plasma lactate in elite canine athletes warrants investigation and determination of their effects on injury rates in dogs is a possible next step in this research. Possible methods to reduce these alterations might include improved maintenance of hydration with readily accessible water during agility exercise, adequate warm-up and cool-down exercise prior to participation in any agility exercise to improve musculoskeletal blood flow, and training methods that increase aerobic muscle capacity. Further understanding of the physiological responses to this type of intense, short-duration exercise may improve performance and reduce injury in dogs participating in this sport.

\section{Competing interests}

The authors declare that they have no competing interests.

\section{Authors' contributions}

WIB carried out the study design, data collection, assay analysis, and drafted the manuscript. AMF designed the timing of the agility course, collected data, and participated in drafting the manuscript. BS performed the assays, standardized the analysis and participated in data interpretation. JJW participated in data collection and interpretation of the data. MEG performed the complete blood count and serum biochemistry analyses, interpreted the findings in these analyses, and participated in writing the conclusions and discussion involving these analyses. ECM participated in data collection, interpretation of the physical examination and cardiopulmonary data and helped to draft the manuscript. All authors read and approved the final manuscript.

\section{Acknowledgements}

The authors wish to thank Jennifer Simpson, Craig Ruaux, Erin Ryan, and the Willamette Agility Group for their assistance. 


\section{Author details}

'Departments of Clinical Sciences (Baltzer, Firshman, Stang, Warnock, McKenzie) and Biomedical Sciences (Gorman), College of Veterinary Medicine, Oregon State University, Corvallis, OR 97331, USA. ${ }^{2}$ Present address: 225 Veterinary Medical Center, 1365 Gortner Ave, St. Paul, MN 55108, USA.

Received: 29 September 2012 Accepted: 19 December 2012 Published: 28 December 2012

\section{References}

1. Agility Association USD: Official Rules and Regulations of United States Dog Agility Association, Inc. In, vol. 1Edited by Gauntt J, Taylor J. Richardson, TX: United States Dog Agility Association, Inc; 2004:71.

2. Levy M, Hall C, Trentacosta N, Percival M: A preliminary retrospective survey of injuries occurring in dogs participating in canine agility. Vet Comp Orthop Traumatol 2009, 22(4):321-324.

3. Rovira S, Munoz A, Benito M: Hematologic and biochemical changes during canine agility competitions. Vet Clin Pathol 2007, 36(1):30-35.

4. Rovira S, Munoz A, Benito M: Fluid and electrolyte shifts during and after Agility competitions in dogs. J Vet Med Sci 2007, 69(1):31-35.

5. Ji LL, Leichtweis $\mathrm{S}$ : Exercise and oxidative stress: sources of free radicals and their impact on antioxidant systems. Age 1997, 20:91-106.

6. McAnulty SR, Hosick PA, McAnulty LS, Quindry JC, Still L, Hudson MB, Dibarnardi AN, Milne GL, Morrow JD, Austin MD: Effect of pharmacological lowering of plasma urate on exercise-induced oxidative stress. Appl Physiol Nutr Metab 2007, 32(6):1148-1155.

7. Mallat Z, Philip I, Lebret M, Chatel D, Maclouf J, Tedgui A: Elevated levels of 8-iso-prostaglandin F2alpha in pericardial fluid of patients with heart failure: a potential role for in vivo oxidant stress in ventricular dilatation and progression to heart failure. Circulation 1998, 97(16):1536-1539.

8. Morrow JD, Roberts $L$ J: The isoprostanes: unique bioactive products of lipid peroxidation. Prog Lipid Res 1997, 36(1):1-21.

9. Rietjens SJ, Beelen M, Koopman R, VANL L, Bast A, Haenen GR: A single session of resistance exercise induces oxidative damage in untrained men. Med Sci Sports Exerc 2007, 39(12):2145-2151.

10. Pilacik B, Nofer TW, Wasowicz W: F2-isoprostanes biomarkers of lipid peroxidation: their utility in evaluation of oxidative stress induced by toxic agents. Int J Occup Med Environ Health 2002, 15(1):19-27.

11. Child R, Brown S, Day S, Donnelly A, Roper H, Saxton J: Changes in indices of antioxidant status, lipid peroxidation and inflammation in human skeletal muscle after eccentric muscle actions. Clin Sci 1999, 96(1):105-115

12. Mastaloudis A, Leonard SW, Traber MG: Oxidative stress in athletes during extreme endurance exercise. Free Radic Biol Med 2001, 31(7):911-922.

13. Hinchcliff KW, Reinhart GA, DiSilvestro R, Reynolds A, Blostein-Fujii A, Swenson RA: Oxidant stress in sled dogs subjected to repetitive endurance exercise. Am J Vet Res 2000, 61(5):512-517.

14. Camus G, Deby-Dupont G, Duchateau J, Deby C, Pincemail J, Lamy M: Are similar inflammatory factors involved in strenuous exercise and sepsis? Intensive Care Med 1994, 20(8):602-610.

15. Barton $M H$, Williamson $L$, Jacks S, Norton N: Effects on plasma endotoxin and eicosanoid concentrations and serum cytokine activities in horses competing in a 48-, 83-, or $159-\mathrm{km}$ endurance ride under similar terrain and weather conditions. Am J Vet Res 2003, 64(6):754-761.

16. McMichael MA, Ruaux CG, Baltzer WI, Kerwin SC, Hosgood GL, Steiner JM, Williams DA: Concentrations of 15F2t isoprostane in urine of dogs with intervertebral disk disease. Am J Vet Res 2006, 67(7):1226-1231.

17. Baltzer WI, McMichael MA, Ruaux CG, Noaker L, Steiner JM, Williams DA: Measurement of urinary 11-dehydro-thromboxane B2 excretion in dogs with gastric dilatation-volvulus. Am J Vet Res 2006, 67(1):78-83.

18. Hollander M, Wolfe DA: Nonparametric Statistical Methods. 2nd edition. New York, New York: John Wiley and Sons, Inc; 1999.

19. Alessio HM, Hagerman AE, Fulkerson BK, Ambrose J, Rice RE, Wiley RL: Lipid and protein oxidation after exhaustive aerobic and isometric exercise. Med Sci Sports Exerc 2000, 32:1576-1581.

20. Piercy RJ, Hinchcliff KW, DiSilvestro RA, Reinhart GA, Baskin CR, Hayek MG, Burr JR, Swenson RA: Effect of dietary supplements containing antioxidants on attenuation of muscle damage in exercising sled dogs. Am J Vet Res 2000, 61(11):1438-1445.

21. Baskin CR, Hinchcliff KW, DiSilvestro R, Reinhart GA, Hayek MG, Chew BP, Burr JR, Swenson RA: Effects of dietary antioxidant supplementation on oxidative damage during prolonged exercise in sled dogs. Am J Vet Res 2000, 61:886-891.

22. Fatouros IG, Chatzinikolaou A, Douroudos II, Nikolaidis MG, Kyparos A, Margonis K, Michailidis Y, Vantarakis A, Taxildaris K, Katrabasas I, et al: Timecourse of changes in oxidative stress and antioxidant status responses following a soccer game. J Strength Cond Res 2010, 24(12):3278-3286.

23. Thomas AC, McLean SG, Palmieri-Smith RM: Quadriceps and hamstrings fatigue alters hip and knee mechanics. J Appl Biomech 2010, 26(2):159-170.

24. Zebis MK, Bencke J, Andersen LL, Alkjaer T, Suetta C, Mortensen P, Kjaer M, Aagaard P: Acute fatigue impairs neuromuscular activity of anterior cruciate ligament-agonist muscles in female team handball players. Scand J Med Sci Sports 2011, 21(6):833-840.

25. Soffler C, Campbell VL, Hassel DM: Measurement of urinary F2isoprostanes as markers of in vivo lipid peroxidation: a comparison of enzyme immunoassays with chas chromatography-mass spectrometry in domestic species. Vet Diagn Invest 2010, 22:200-209.

26. Schuster DP, Kozlowski JK, McCarthy T, Morrow J, Stephenson A: Effect of endotoxin on oleic acid lung injury does not depend on priming. J Appl Physiol 2001, 91(5):2047-2054

27. Walker TG, Chan DL, Freeman LM, Milbury PE, Blumberg JB: Serial determination of biomarkers of oxidative stress and antioxidant status in dogs with naturally occuring gastric dilatation-volvulus. J Vet Emerg Crit Care 2007, 17(3):250-256

28. Urso ML, Clarkson PM: Oxidative stress, exercise, and antioxidant supplementation. Toxicology 2003, 189(1-2):41-54.

29. Pasquini A, Luchetti $E$, Cardini G: Evaluation of oxidative stress in hunting dogs during exercise. Res Vet Sci 2010, 89(1):120-123.

30. Munoz A, Riber C, Santisteban R, Aquera El, Castejon FM: Cardiovascular and metabolic adaptations in horses competing in cross-country events. J Vet Med Sci 1999, 59:747-750.

31. Gaesser GA, Poole DC: Lactate and ventilatory thersholds: disparity in time course of adaptations to training. J Appl Physiol 1986, 61:999-1004.

32. Rose RJ, Boomberg MS: Responses to sprint exercise in the greyhound: effects on haematology, serum biochemistry and muscle metabolites. Res Vet Sci 1989, 47:212-218.

33. Nold $J$, Peterson $L$, Fedde MR: Physiological changes in the running greyhound (canis domesticus): influence of race length. Comp Biochem physiol 1991, 100A(3):623-627.

34. pieschl RL, Toll PW, Leith DE, Peterson LJ, Fedde MR: Acid-base changes in the running greyhound: contributing variables. J Appl Physiol 1992, 73(6):2297-2304

35. Hill RC, Lewis DD, Scott KC, Omori M, Jackson M, Sundstrom DA, Jones GL, Speakman JR, Doyle CA, Butterwick RF: Effect of increased dietary protein and decreased dietary carbohydrate on performance and body composition in racing Greyhounds. Am J Vet Res 2001, 62(3):440-447.

36. Steiss J, Ahmad HA, Cooper P, Ledford C: Physiologic responses of healthy Laborador retrievers during field trial training and competition. J Vet Intern Med 2004, 18:147-151.

37. Steiss JE, Wright JC: Respiratory alkalosis and primary hypocapnia in Laborador retrievers participating in field trials in high-ambienttemperature conditions. Am J Vet Res 2008, 69(10):1262-1267.

38. Matwichuck CL, Taylor S, Shmon CL, Kass PH, Shelton GD: Changes in rectal temperature and hematologic, biochemical, blood gas, and acid-base values in healthy labrador Retrievers before and after strenuous exercise. Am J Vet Res 1999, 60(1):88-92.

39. Guy PS, Snow DH: Skeletal muscle fibre composition in the dog and its relationship to athletic ability. Res Vet Sci 1981, 31:244-248.

40. Essen B, Jansson E, Henriksson J, Taylor AW, Saltin B: Metabolic characteristics of fibe types in human skeletal muscle. Acta Physio/ Scand 1975, 95(2):153-165.

41. Saltin B: Metabolic fundamentals in exercise. Med Sci Sports 1973, 5(3):137-146.

42. Snow DH, Guy PS: Muscle fibre type composition of a number of limb muscles in different types of horse. Res Vet Sci 1980, 28(2):137-144.

43. Evans DL, Harris RC, Snow DH: correlation of racing performance with blood lactate and heart rate after exercise in thoroughbred horses. Equine Vet J 1993, 25(5):441-445.

44. Bishop D, Jenkins DG, McEniery M, Carey MF: Relationship between plasma lactate parameters and muscle characteristics in female cyclists. Med Sci Sports Exerc 2000, 32(3):1088-1093. 
45. Kuwahara H, Horie T, Ishikawa S, Tsuda C, Kawakami S, Noda Y, Kaneko T, Tahara S, Tachibana T, Okabe M, et al: Oxidative stress in skeletal muscle causes severe disturbance of exercise activity without muscle atrophy. Free Radic Biol Med 2010, 48(9):1252-1262.

46. Reid MB: Free radicals and muscle fatigue: Of ROS, canaries, and the IOC. Free Radic Biol Med 2008, 44(2):169-179.

47. Reid MB, Haack KE, Franchek KM, Valberg PA, Kobzik L, West MS: Reactive oxygen in skeletal muscle. I. Intracellular oxidant kinetics and fatigue in vitro. J Appl Physiol 1992, 73(5):1797-1804

48. Kelly MK, Wicker RJ, Barstow TJ, Harms CA: Effects of N-acetylcysteine on respiratory muscle fatigue during heavy exercise. Respir Physiol Neurobiol 2009, 165(1):67-72

49. Murphy KT, Medved I, Brown MJ, Cameron-Smith D, McKenna MJ: Antioxidant treatment with $\mathrm{N}$-acetylcysteine regulates mammalian skeletal muscle $\mathrm{Na}+-\mathrm{K}+-\mathrm{ATPase}$ alpha gene expression during repeated contractions. Exp Physiol 2008, 93:1239-1248.

50. Lindinger MI, McKelvie RS, Heigenhauser GJF: K+ and Lac- distribution in humans during and after high-intensity exercise: role in muscle fatigue attenuation? J Appl Physiol 1995, 78(3):765-777.

51. Yoshikawa T, Mori S, Santiesteban AJ, Sun TC, Hafstad E, Chen J, Burr DB: The effects of muscle fatigue on bone strain. J Exp Biol 1994, 188:217-233.

52. Milgrom C, Radeva-Petrova DR, Finestone A, Nyska M, Mendelson S, Benjuya $\mathrm{N}$, Simkin A, Burr D: The effect of muscle fatigue on in vivo tibial strains. J Biomech 2007, 40:845-850.

53. Chamberlain KG, Tong M, Penington DG: Properties of the exchangeable splenic platelets released into the circulation during exercise-induced thrombocytosis. Am J Hematol 1990, 34(3):161-168.

54. Schmidt KG, Rasmusson JW: Exercise induced change in the vivo distribution of in-labeled platelets. Scand J Haematol 1984, 32:159-166.

55. Ahmadizad S, El-Sayed MS, Maclaren DP: Responses of platelet activation and function to a single bout of resistance exercise and recovery. Clin Hemorheol Microcirc 2006, 35(1-2):159-168.

56. Ilkiw JE, Davis PE, Church DB: Hematologic, biochemical, blood-gas, and acid-base values in Greyhounds before and after exercise. Am J Vet Res 1989, 50:583-586.

57. Brun JF: Exercise hemorheology as three acts play with metabolic actors: Is it of clinical relevance? Clin Hemorheol Microcirc 2002, 26:155-174.

58. Brun JF, Khaled S, Raynaud E, Bouix D, Micallef JP, Orsetti A: The triphasic effects of exercise on blood rheology: which relevance to physiology and pathophysiology? Clin Hemorheol Microcirc 1998, 19(2):89-104.

59. Ernst E, Daburger L, Saradeth T: The kinetics of blood rheology during and after prolonged standardized exercise. Clin Hemorheol 1991, 11:429-439.

60. Mitten LA, Hinchcliff KW, Pate JL, Kohn CW, McKeever KH: Effect of exercise intensity on plasma prostaglandin concentrations in horses. Am J Vet Res 1995, 56(1):122-126.

61. Todd MK, Goldfarb AH, Boyer BT: Effect of exercise intensity on 6-ketoPGF1 alpha, TXB2, and 6-keto-PGF1 alpha/TXB2 ratios. Thromb Res 1992, 65(4-5):487-493

62. Catella F, Reilly MP, Delanty N, Lawson JA, Moran N, Meagher E, FitzGerald GA: Physiological formation of 8-epi-PGF2 alpha in vivo is not affected by cyclooxygenase inhibition. Adv Prostaglandin Thromboxane Leukot Res 1995, 23:233-236.

63. Chiabrando C, Rivoltella L, Alberti E, Bonollo M, Djurup R, Fanelli R: Urinary excretion and origin of 11-dehydro-2,3-dinor-thromboxane B2 in man. Prostaglandins 1993, 45(5):401-411.

doi:10.1186/1746-6148-8-249

Cite this article as: Baltzer et al:: The effect of agility exercise on eicosanoid excretion, oxidant status, and plasma lactate in dogs. BMC Veterinary Research 2012 8:249.

\section{Submit your next manuscript to BioMed Central and take full advantage of:}

- Convenient online submission

- Thorough peer review

- No space constraints or color figure charges

- Immediate publication on acceptance

- Inclusion in PubMed, CAS, Scopus and Google Scholar

- Research which is freely available for redistribution

Submit your manuscript at www.biomedcentral.com/submit
Ciomed Central 